\title{
Prevalence of Toxoplasma gondii infection in household and feral cats in Korea
}

\author{
Sung-Eon Kim ${ }^{1,2} \cdot$ Ran Choi $^{1,2} \cdot$ Seung-Won Kang ${ }^{3} \cdot$ Changbaig Hyun $^{2}(\mathbb{D}$
}

Received: 1 July 2015/ Accepted: 9 February 2017/Published online: 16 February 2017

(C) Indian Society for Parasitology 2017

\begin{abstract}
This study was designed to investigate the prevalence rate of Toxoplasma gondii (T. gondii) infection in household cats in Korea. One hundred household cats and 50 feral cats from nine of the largest cities in Korea were enrolled in this study. The tests performed in this survey was an in-house rapid screen IgG and IgM combo test, faecal PCR test for T. gondii oocysts, and an ELISA immunoassay for IgG antibodies. There were no household cats positive for $T$. gondii infection detected using the inhouse $\operatorname{IgG}$ and IgM rapid screen combo test, although 6/50 and $0 / 50$ feral cats were positive in $\operatorname{IgG}$ and IgM tests, respectively. This initial finding was confirmed by subsequent ELISA test for IgG antibody and PCR for $T$. gondii in faeces. Despite the higher prevalence rate of the disease in feral cats in Korea, we did not find any household cats that were either infected or exposed previously to $T$. gondii in our study population. Our study indicates that there is minimal risk of $T$. gondii transmission from household cats to human in Korea.
\end{abstract}

Keywords Toxoplasma gondii - Infection · Transmission · Zoonosis · Cat

Changbaig Hyun

hyun5188@kangwon.ac.kr

1 Dasom Animal Hospital, Busan, Korea

2 Section of Small Animal Internal Medicine, College of Veterinary Medicine and Institute of Veterinary Science, Kangwon National University, Chuncheon, Korea

3 Animal, Plant and Fisheries Quarantine and Inspection Agency, Anyang, Korea

\section{Introduction}

Toxoplasmosis is an important zoonotic disease caused by Toxoplasma gondii (T. gondii) reported in almost all domestic animals and humans but subclinical asymptomatic disease in animals is far more common than symptomatic clinical disease (Dubey 2009). Since cats are the final host for $T$. gondii, the prevalence of $T$. gondii in feral cats has been widely investigated in many countries (Maruyama et al. 2003; Miró et al. 2004; Haddadzadeh et al. 2006; Zhang et al. 2009). Although most transmission to humans is most likely due to eating undercooked infected meat (e.g., lamb and pork) and/or unwashed fruits and vegetables, many doctors believe erroneously that an infected cat is more likely the route of transmission of $T$. gondii to humans. In fact, the prevalence of $T$. gondii in feral cats is relatively high in some countries (Maruyama et al. 2003; Haddadzadeh et al. 2006; Zhang et al. 2009). In Korea, several studies have also found a relatively high prevalence rate (13-45\%) of T. gondii in feral cats (Kim et al. 2008; Lee et al. 2008). Despite high prevalence rate in feral cats, it is hardly to see clinically ill cats by toxoplasmosis in local veterinary practice in Korea. Therefore this study focused on determining the actual prevalence rate of $T$. gondii in household cats from nine major cities across Korea.

\section{Materials and methods}

\section{Cats}

One hundred household cats (50 females, 50 males, 1-12 years old, $3.4-7.2 \mathrm{~kg}$ in body weight) and 50 feral cats ( 25 females, 25 males, unidentified age, $2.4-8.1 \mathrm{~kg}$ in 
body weight) from nine major cities across Korea were enrolled in this survey. These cats were clinically healthy and visited local veterinary clinics for either vaccination or routine health check. All feral cats were presented for trapneutering-release program and were clinically healthy. We obtained the consent of the owners and administration of cat shelter for sample collection and publication of this survey, prior to the study.

\section{Blood collection and assays}

Blood was collected from each cat via a cephalic or jugular puncture. The blood was placed into a serum separation tube and centrifuged for $5 \mathrm{~min}$ at $1800 \mathrm{~g}$ after clotting at room temperature for $2 \mathrm{~h}$. One to two drops of serum were transferred to the rapid screen test kit for IgG/IgM antibodies against $T$. gondii (SensPERT Feline Toxo IgG/IgM $\mathrm{Ab}$ Test Kit, Vetall, Korea). The test was performed according to the manufacturer's instructions. The ELISA test against $T$. gondii IgG antibody was performed according to Choi et al. (1992). Faecal samples from all cats were also collected to detect $T$. gondii oocysts from faeces. DNA extraction from faecal samples was performed using a DNeasy Mini column kit (Qiagen, Hilden, Germany) according to the manufacturer's instructions. PCR test against $T$. gondii oocysts were performed according to Herrmann et al. (2011).

\section{Results}

No household cats were found positive using the rapid screen IgG and IgM combo test. The ELISA test against IgG antibody of $T$. gondii also found no positive cats and confirmed the initial screen test. Further PCR test against $T$. gondii oocysts also found no positive cats. In feral cats, $6 / 50$ cats ( 3 females and 3 males) were positive for $\operatorname{IgG}$ test, while no cats were positive for IgM test. Further ELISA test against $\operatorname{IgG}$ antibody of $T$. gondii confirmed seropositive in 6 cats which were previously positive in rapid screen IgG test. However, PCR test against $T$. gondii oocysts failed to find positive cats.

\section{Discussion}

Several epidemiological surveys conducted in several countries found a high prevalence of $T$. gondii infections in feral cats, with a substantially lower rate in household cats (Maruyama et al. 2003; Miró et al. 2004; Haddadzadeh et al. 2006; Zhang et al. 2009). Similarly, the prevalence rate of $T$. gondii in feral cats in Korea was also found to be relatively high in general (13-45\%; Kim et al.
2008; Lee et al. 2008). In contrast, a recent survey in one metropolitan area of Seoul, found no seropositive cats (Lee et al. 2010). This previous report is corroborated by our study where we did not detect any seropositive household cats, although $6 / 50$ feral cats were seropositive (12\%). Furthermore, we did not detect any cats shedding T. gondii oocysts in 100 household cats and 50 feral cats derived from nine major cities in Korea. The prevalence rate in feral cats in our study was similar to other study (Kim et al. 2008). All positive cats were from southern part of Korea, where annual mean temperature is higher than other regions. Probably, warmer environment might be a contributing factor for $T$. gondii infection. There was no sex predilection for $T$. gondii infection in this study. According to survey reports from other countries, the prevalence rate of $T$. gondii in household cats was $25.5 \%$ in Spain (Miró et al. 2004), 36\% in Iran (Haddadzadeh et al. 2006), $17.9 \%$ in China (Zhang et al. 2009), and $5.4 \%$ in Japan (Maruyama et al. 2003). Furthermore, the prevalence rate of $T$. gondii in feral cats was higher than that of household cats in the same area, as observed in our study.

Authors believed several factors involved zero percentage of prevalence rate of $T$. gondii in household cats in this study. Firstly, the household cats had better health care, and were raised in a better hygienic environment, so that the risk of exposure to $T$. gondii might be lower. Secondly, the preference of commercial diets by Korean cat owners might also reduce the risk of food contamination from $T$. gondii, because the major sources of transmission of $T$. gondii in humans are undercooked infected meats and unwashed vegetables. Unlike raw meats and vegetables, the commercial diets including dry pellets and wet cans tend to be better sterilized prior to the sales. Therefore the risk of exposure to $T$. gondii might also be lower in household cats for the reasons stated previously. Lastly, unlike other countries, most household cats are strictly indoor cats, so the risk of exposure to infected feral cats is reduced considerably. This assumption is supported by the lower prevalence rate of $T$. gondii in household cats in Japan, where the living environment is much similar to Korea.

The importance of this study is that we have found a zero prevalence of $T$. gondii in household cats from nine major cities in Korea. This outcome suggests that the mode of transmission of $T$. gondii to humans is less likely to be via the household cat. Furthermore, our study also suggests that the risk of transmission of $T$. gondii from the cats to humans can be dramatically reduced if the cat lives indoors and fed uncontaminated food.

Acknowledgements This study was supported by Kangwon National University. 


\section{References}

Choi WY, Nam HW, Youn JH, Kim DJ, Kong Y, Kang SY, Cho SY (1992) Detection of antibodies in serum and cerebrospinal fluid to Toxoplasma gondii by indirect latex agglutination test and enzyme-linked immunosorbent assay. Korean J Parasitol 30:83-90

Dubey JP (2009) Toxoplasmosis in sheep-the last 20 years. Vet Parasitol 163(1-2):1-14

Haddadzadeh HR, Khazraiinia P, Aslani M, Rezaeian M, Jamshidi S, Taheri M, Bahonar A (2006) Seroprevalence of Toxoplasma gondii infection in stray and household cats in Tehran. Vet Parasitol 138(3-4):211-216

Herrmann DC, Maksimov A, Pantchev N, Vrhovec MG, Conraths FJ, Schares G (2011) Comparison of different commercial DNA extraction kits to detect Toxoplasma gondii oocysts in cat faeces. Berl Munch Tierarztl Wochenschr 124(11-12):497-502

Kim HY, Kim YA, Kang SW, Lee HS, Rhie HG, Ahn HJ, Nam HW, Lee SE (2008) Prevalence of Toxoplasma gondii in stray cats of Gyeonggi-do, Korea. Korean J Parasitol 46(3):199-201
Lee JY, Lee SE, Lee EG, Song KH (2008) Nested PCR-based detection of Toxoplasma gondii in German shepherd dogs and stray cats in South Korea. Res Vet Sci 85(1):125-127

Lee SE, Kim JY, Kim YA, Cho SH, Ahn HJ, Woo HM, Lee WJ, Nam HW (2010) Prevalence of Toxoplasma gondii infection in stray and household cats in regions of Seoul, Korea. Korean J Parasitol 48(3):267-270

Maruyama S, Kabeya H, Nakao R, Tanaka S, Sakai T, Xuan X, Katsube Y, Mikami T (2003) Seroprevalence of Bartonella henselae, Toxoplasma gondii, FIV and FeLV infections in domestic cats in Japan. Int J Parasitol 33(13):1525-1535

Miró G, Montoya A, Jiménez S, Frisuelos C, Mateo M, Fuentes I (2004) Prevalence of antibodies to Toxoplasma gondii and intestinal parasites in stray, farm and household cats in Spain. Vet Parasitol 126(3):249-255

Zhang H, Zhou DH, Zhou P, Lun ZR, Chen XG, Lin RQ, Yuan ZG, Zhu XQ (2009) Seroprevalence of Toxoplasma gondii infection in stray and household cats in Guangzhou, China. Zoonoses Public Health 56(9-10):502-505 